\title{
'Isolated' germline mosaicism in the phenotypically normal father of a girl with X-linked hypophosphatemic rickets
}

\section{Yunting Lin', Yanna Cai', Jianan Xu', Chunhua Zeng', Huiying Sheng', Yang Yư ${ }^{2}$, Xiuzhen Li' ${ }^{1}$ and Li Liu' ${ }^{1}$}

${ }^{1}$ Department of Genetics and Endocrinology and 2Medical Reproduction Center, Guangzhou Women and Children's Medical Center, Guangzhou Medical University, Guangzhou, China
Correspondence should be addressed to L Liu Email

liliuchina@qq.com

\begin{abstract}
Objective: X-linked hypophosphatemic rickets (XLHR) is the most common form of inherited rickets caused by pathogenic variants of PHEX gene with an X-linked dominant inheritance pattern. Precise molecular diagnosis of pathogenic variant will benefit the genetic counseling and prenatal diagnosis for the family with XLHR. Here, we presented an 'isolated' germline mosaicism in the phenotypically normal father of a girl with XLHR.

Methods and results: For the initial molecular screen of PHEX gene, DNA samples of the proband and her parents were extracted from their peripheral blood samples respectively. Sanger sequencing found a 'de novo' novel heterozygous variant, c.1666C>T(p.Q556X), at the PHEX gene in the proband, but not in her phenotypically healthy parents. Due to an occasional abnormality of his serum phosphate previously, further examinations for the father were taken to exclude the possibility of paternal mosaicism. Eight samples from different tissues were analyzed for PHEX gene by Sanger sequencing. Surprisingly, one 'isolated' germline mosaicism was detected only in his sperm with an estimated frequency of $26.67 \%$. The mosaic allele was identical to the c.1666C > T(p.Q556X) variant in the proband.

Conclusions: This is the first case of 'isolated' germline mosaicism with pathogenic PHEX variant. Our study provides accurate diagnosis and valuable counseling for this family. This report also alerts clinicians and geneticists to exclude the possibility of the isolated germline mosaicism and prevent intrafamilial recurrences of inherited diseases.
\end{abstract}

\section{Introduction}

$\mathrm{X}$-linked hypophosphatemic rickets (XLHR) is a rare monogenic disease caused by the loss-of-function variants in the PHEX gene located on Xp22.11 with an X-linked dominant inheritance pattern. Accounting for over $80 \%$ of familial cases and about $70 \%$ of sporadic cases, XLHR is the most common form of inherited rickets with the estimated prevalence ranging from $1 / 60000$ to $1 / 20000$ $(1,2,3,4,5,6,7,8,9)$.

Occurring as a consequence of chaotic phosphate metabolism and defective bone mineralization, XLHR patients fail to thrive and present lower extremity deformities, osteoporosis, bone pain, and poor skeletal and dental development with a biochemical manifestation of hypophosphatemia $(1,2)$.

As most of XLHR patients begin to present clinical manifestations within the first year of life, early diagnosis and treatment could minimize bone mineralization disturbances, correct rachitic symptoms, and improve growth and development velocity to some extent (2, 10, 11). Particularly, precise molecular diagnosis with pathogenic variant will benefit the genetic counseling and prenatal diagnosis for the family with inherited diseases.

However, deleterious variant mosaicism especially symptomless isolated germline mosaicism will challenge and

Published by Bioscientifica Ltd. 
obstruct the accurate counseling and efficient prevention $(12,13,14)$. Here we described an unusual XLHR pedigree with an 'isolated' germline mosaicism father of PHEX gene.

\section{Patients and methods}

\section{Patients}

The family of a girl with XLHR (Fig. 1A) was enrolled in Guangzhou Women and Children's Medical Center. All the subjects were of Han ethnicity.

Physical and X-ray examinations were performed by specialist physicians and radiologists, respectively. Family history was inquired by genetic counselors. Whole blood, serum and urine samples were used to detect relevant biomedical and hormonal indexes in the hospital's clinical laboratory center. In particular, serum FGF23 levels were measured using the human FGF23 ELISA Kit against Tyr25-Ile251 (Arg179Gln) (FineTest, Wuhan, China).

This study was approved by the Institutional Review Board of Guangzhou Women and Children's Medical

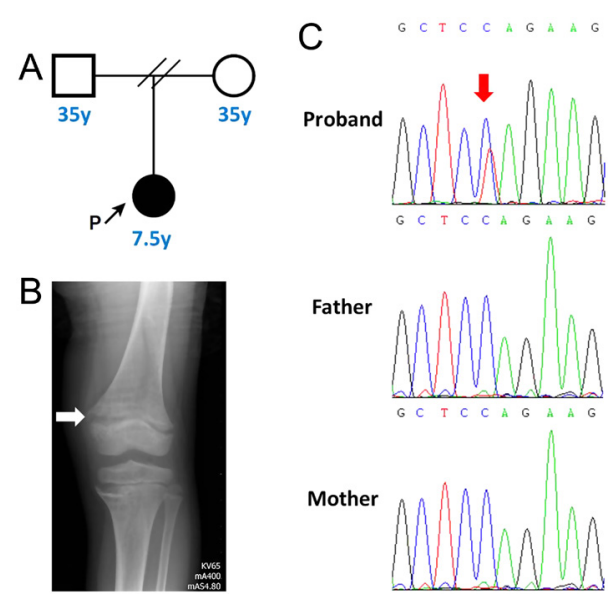

\section{Figure 1}

The pedigree, radiological manifestations and sequencing diagrams of the XLHR family. (A) The family pedigree. The arrow indicates the proband. Filled symbol represents the individual with clinical symptoms, while clear symbol is for unaffected family member. (B) The proband's X-ray shows typical rachitic radiological signs characterized by a general decrease in bone density, diffuse bony contour, widened and distorted metaphyses along with blurred epiphyseal line, coarse axial skeleton trabeculation, and fuzzy alterations in the zone of provisional calcification in the growth plate (the white arrow). (C) The Sanger sequencing chromatograms of the entire family. The red arrow indicates the variant site. A full colour version of this figure is available at https://doi.org/10.1530/EJE-19-0472.
Center (Guangzhou, China). The informed consents were obtained from all subjects or their guardians (for the under-aged participants).

\section{Mutational screening of PHEX gene}

gDNA was extracted from whole blood samples using DNeasy Blood \& Tissue Kit (QIAGEN). The entire coding regions of PHEX gene, exonic sequences together with adjoining intronic boundaries (NG_007563.2, NM_000444.5), were amplified by PCR using 22 pairs of PHEX-specific primers designed by Primer Premier 5 software. The PCR products were detected by agarose gel electrophoresis and directly sequenced with an ABI 3730xl DNA Analyzer. The sequencing chromatograms were read by Chromas software, while the exported sequences were aligned with the reference sequence using DNAMAN software.

All the captured variants were verified by Sanger sequencing with both forward and reverse primers on two independent PCR products. The SNP databases, including 1000Genomes, ESP6500, ExAC and dbSNP, were employed to exclude the polymorphic alleles, whilst the PHEX mutation databases, HGMD and PHEXdb, were engaged to screen the known pathogenic variants. For novel variants absent from the above SNP and PHEX mutation databases, in-silico analyses with the online tools of PROVEAN, SIFT, PolyPhen-2, MutationTaster, MutationAssessor and FATHMM were implemented.

\section{Mosaic status survey in multiple tissues from the father}

Eight different samples were collected from the father, including hair (principally for hair roots), oral epithelium (obtained by buccal swab), saliva (mixture of exfoliated oral epitheliums and a small amount of leukocytes from salivary glands), nail, cuticle, whole blood, urine (including renal and bladder exfoliated epithelial cells), and sperm. gDNA was extracted by standard or modified procedure of DNeasy Blood \& Tissue Kit (QIAGEN) and analyzed for PHEX gene as described above, respectively.

\section{Results}

\section{The clinical and genetic features of the proband}

The proband began to present genu varum when she was able to walk at 1 year of age and was diagnosed as 
hypophosphatemic rickets (HR) by Children's Hospital of Shanghai at 2 years of age.

She first presented at our clinic at 7.5 years of age. Physical examination showed short stature $(<-2$ s.D. $)$ (Table 1), apparent leg deformities (genu varum), and bracelet. X-ray examination revealed typical rachitic radiological signs (Fig. 1B). Renal tubular acidosis was first ruled out by the normal values of blood potential of hydrogen $(\mathrm{pH})$, bicarbonate (HCO3-), base excess of blood (BE-B) and extracellular fluid (BE-ECF) (data not shown). Serum biochemical tests indicated a strikingly decreased level of serum phosphate in combination with elevated alkaline phosphatase (ALP), normal calcium and 1,25(OH)2 vitamin D3 concentration. The level of serum parathyroid hormone (PTH) was normal (Table 1). Thus, the diagnosis of HR was also made based on these physical, radiological and laboratorial results, though the serum fibroblast growth factor 23 (FGF23), tubular reabsorption of phosphate (TRP) and the ratio of tubular maximum reabsorption rate of phosphate to glomerular filtration rate (TmP/GFR) were normal (Table 1).

As XLHR is the most frequent form of inherited rickets, the proband was initially subjected to DNA analysis of PHEX gene. As expected, a novel c.1666C >T(p.Q556X) hererozygous variant at the exon 16 was found in the proband, whereas absent in her disease-free parents using gDNA from whole blood samples, indicating a 'de novo' pattern (Fig. 1C). Giving rise to a premature termination of translation and producing a truncated polypeptide, the c.1666C>T(p.Q556X) variant was assessed as a

Table 1 Growth details and laboratory indexes of the proband at 7.5 years of age.

\begin{tabular}{|c|c|c|}
\hline Item & Value & Reference range \\
\hline Weight (kg) & 21 & 18.1 (-2 S.D.) \\
\hline Length $(\mathrm{cm})$ & $114.1 \downarrow$ & 115.4 (-2 s.D.) \\
\hline Phosphate (mmol/L) & $0.84 \downarrow$ & $1.29-1.94$ \\
\hline Calcium (mmol/L) & 2.48 & $2.19-2.69$ \\
\hline ALP (U/L) & $765 \uparrow$ & $118-390$ \\
\hline 1,25(OH)2 vitamin D3 (nmol/L) & 56.2 & $30.0-100.0$ \\
\hline PTH (pmol/L) & 3.46 & 1.2-7.1 \\
\hline FGF23 (pg/mL) & 31 & $0-100$ \\
\hline $\operatorname{TRP}(\%)$ & 92.3 & 85-95 \\
\hline TmP/GFR (mmol/L) & 0.92 & $0.80-1.35$ \\
\hline
\end{tabular}

ALP, alkaline phosphatase; PTH, parathyroid hormone; FGF23, fibroblast growth factor 23, the average FGF23 level in 10 healthy girls at age of 6-9 years is $38.55 \pm 56.31 \mathrm{pg} / \mathrm{mL}$ (range $=0-134.4 \mathrm{pg} / \mathrm{mL}$ ) in this study; TRP, tubular reabsorption of phosphate, which is calculated according to the formula of (1 - (urine phosphate $\times$ serum creatinine)/(serum phosphate $\times$ urine creatinine) $) \times 100 \%$; TmP/GFR, the ratio of tubular maximum reabsorption rate of phosphate to glomerular filtration rate, which is derived based on the nomogram of renal threshold phosphate concentration (15). deleterious variant, revealing a definite diagnosis of XLHR for the proband.

\section{The 'isolated' germline mosaicism father}

During follow-up of the proband, her father recalled an occasional abnormality of his serum phosphate several years ago. He presented his eagerness for second child and required to exclude the possibility of next pregnancy with XLHR.

Routine physical, radiological and laboratory examinations of XLHR were further carried out for him, but no obvious abnormity was found (Fig. 2A, B and Table 2).

As our in-house study showed the pathogenic PHEX variants was transmitted from the affected parents in $46.67 \%$ XLHR pediatric patients (21/45), and a healthy mother with somatic and germline mosaicism of IPEX syndrome was previously found in our laboratory (14), it may be possible that the deleterious PHEX variant of this proband was delivered from the father if he was a mosaicism.

To ascertain the possibility that the father was a mosaicism, eight different tissues of the father were collected for PHEX gene analysis. In details, hair, oral epithelium, saliva, nail and cuticle were stemmed from ectoderm, while whole blood, urine, and sperm were mesoderm derived.

Consistent with the above gene result, no detrimental variant at the exon 16 of PHEX gene was displayed in hair, oral epithelium, saliva, nail, cuticle, whole blood and urine. However, to our surprise, an apparently $\mathrm{T}$ peak besides the wild-type $\mathrm{C}$ allele was present at the c.1666 nucleotide site in gDNA from sperm (Fig. 2C). As only the sperm among eight investigated tissues shared the same deleterious variant of PHEX gene to the proband, the father could be regarded as a probably 'isolated' germline mosaicism, even many other tissues remained to be analyzed.

\section{Discussion}

The classical hypothesis of XLHR is that the deficiencies of the PHEX gene lead to an excess of FGF23, a circulating phosphaturic hormone, which suppresses the TRP, subsequently contributes to hypophosphatemia, and results in impaired bone mineralization finally $(2,16,17$, $18,19,20)$.

In this study, the FGF23 level, TRP and TmP/GFR are normal in the proband. Indeed, increased FGF23 levels 

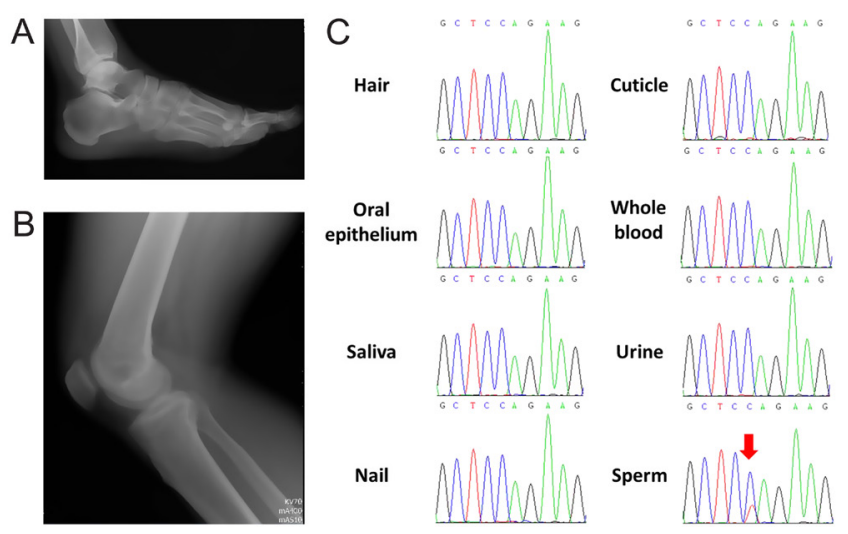

\section{Figure 2}

The radiological manifestations and sequencing diagrams of the father. ( $A$ and $B$ ) X-ray presents a normal radiological phenotype of the father. (C) The Sanger sequencing chromatograms of the father. The red arrow indicates the variant site. A full colour version of this figure is available at https://doi.org/10.1530/EJE-19-0472.

and decreased TRP have been presented in some, but not all XLHR patients $(8,21,22,23,24,25,26)$, indicating that the correlation between PHEX and FGF23 is still unclear, the diagnostic value of FGF23 and TRP in XLHR is limited, and the exact mechanism of this disease remains to be further explored.

As is known, mosaicism is a condition that tissues or cells within the same organism have different genetic makeup. A mosaicism individual with pathogenic variants at a disease gene may develop symptoms or not depending on how the tissues and cells are involved. This condition may interfere molecular diagnosis using blood samples conventionally, if the blood cells are not affected.

XLHR is a dominant disease, and the somatic mosaicism also shows clinical symptoms but mildly, which reminds us to investigate the disease-causing

Table 2 Growth details and laboratory indexes of the father at 35 years of age.

\begin{tabular}{|c|c|c|}
\hline Item & Value & Reference range \\
\hline Length $(\mathrm{cm})$ & 169 & 166.6 (-1 S.D.) \\
\hline Phosphate (mmol/L) & 1 & $0.85-1.51$ \\
\hline Calcium (mmol/L) & 2.41 & $2.11-2.52$ \\
\hline $\operatorname{ALP}(U / L)$ & 75 & $45-125$ \\
\hline 1,25(OH)2 vitamin D3 (nmol/L) & 38 & $30.0-100.0$ \\
\hline PTH (pmol/L) & 2.58 & $1.2-7.1$ \\
\hline FGF23 (pg/mL) & 0 & $0-100$ \\
\hline TRP (\%) & 97.6 & 85-95 \\
\hline TmP/GFR (mmol/L) & 1.34 & $0.80-1.35$ \\
\hline
\end{tabular}

The average FGF23 level in 14 healthy men at age of $30-40$ years is $1.83 \pm 5.11 \mathrm{pg} / \mathrm{mL}$ (range $=0-18.2 \mathrm{pg} / \mathrm{mL}$ ) in this study. variant using other tissue samples. To date, four somatic mosaicism male patients with XLHR have been reported $(21,27,28,29)$, and our in-house study also identifies two XLHR mosaic boys carrying heterozygous PHEX pathogenic variants.

However, in extreme instances, isolated germline mosaicism without a visible phenotype will be omitted. The transmission of isolated germline mosaic alleles from a phenotypically normal parent may be misjudged as ' $d e$ novo' pattern, challenging the evaluation of recurrent risk and the implement of essential prevention.

In this study, the clinical and molecular diagnosis of the proband was definite; however, her father with normal phenotype and 'normal' genotype at her PHEX variant site in the DNA from peripheral blood samples orally recounted an occasional abnormality of his serum phosphate several years ago at another hospital.

Therefore, we had further evaluated routine physical, radiological and laboratory indexes of XLHR for him, but no obvious abnormity was found at our hospital. For this inconformity, there may be several possibilities: (1) the previous serum phosphate was mildly decreased which might be a physiological fluctuation; (2) the serum phosphate was misremembered by the father; (3) the father is a somatic and germline mosaicism, and so forth. As that report with 'abnormal' serum phosphate was lost, we did not obtain the detailed value and determine which possibility is true. According to the examinations at our hospital, we consider that the father is asymptomatic.

Despite that, we still investigate more different tissues for him. In terms of minimal injury, we collected eight obtainable samples. The healthy father was identified with the identical pathogenic PHEX variant to his daughter only in the sperm, whereas other involved tissues, including hair, oral epithelium, saliva, nail, cuticle, whole blood and urine, were wild-type.

For the sensitivity and accurate frequency of a mosaic allele, next-generation sequencing (NGS) with high depth is more preferred than Sanger sequencing (30). However, the father's limited samples are not enough for NGS. In addition, in our experience, a mosaic allele could be identified as low as $1.72 \%$ by Sanger sequencing (14), supporting the reliability of this study.

As the available clinical and genetic evidence did not indicate that the father is a somatic mosaicism, we speculated that he is a probably 'isolated' germline mosaicism. However, we could not totally exclude the possibility that the father is a somatic and germline mosaicism as many other tissues are not involved. 
Actually, this 'isolated' germline mosaicism father might be missed if he did not recall a previous occasional abnormality of his serum phosphate, and if we did not investigate more different tissues as his physical, X-ray and laboratory results were normal. It is an accidental event beyond our expectation.

Additionally, as the father was confirmed as a germline PHEX mosaicism of c.1666C>T(p.Q556X) variant, an approximately $26.67 \%$ recurrence risk of XLHR upon his progenies was estimated according to the $\mathrm{T}$ allele frequency in sperm (Fig. 2C). Reviewing his mosaic status, we speculated a spontaneous mutagenesis during the late embryonic development.

In conclusion, this study describes an 'isolated' germline mosaicism case with pathogenic PHEX variant for the first time. Our study provides accurate diagnosis of pathogenic variant and inheritance pattern, predicts the timing of somatic mutational event, and addresses valuable genetic counseling of recurrent risk for this family, especially for this father. Moreover, this accidental 'isolated' germline mosaicism case with normal phenotype opens the possibility on the genetic basis of diseases. Our study also alerts clinicians and geneticists to be cautious about the isolated germline mosaicism to avoid missing diagnosis.

\section{Declaration of interest}

The authors declare that there is no conflict of interest that could be perceived as prejudicing the impartiality of this case report.

\section{Funding}

This work was supported by the National Natural Science Foundation of China (grant number 81701128); the Natural Science Foundation of Guangdong Province, China (grant number 2016A030313500); and the fund from Guangzhou Institute of Pediatrics/Guangzhou Women and Children's Medical Center (grant number Pre-NSFC-2019-008).

\section{Author contribution statement}

All the listed authors were involved in drafting or editing this article, and approved its submission and publishment. $L \mathrm{~L}$ and $Y \mathrm{~L}$ conceived and designed the study. $L L, C Z, Y Y$ and $X L$ recruited the family, performed physical examinations, and collected their medical history and samples. $Y$ $L, Y C, J X$ and $\mathrm{H}$ S performed the experiments and acquired the data. $Y \mathrm{~L}$ analyzed the data. $L \mathrm{~L}, \mathrm{Y} \mathrm{L}$ and $\mathrm{C} Z$ wrote the paper.

\section{Acknowledgements}

The authors would like to thank the enrolled family for participation in this study. They also thank the Department of Radiology and Clinical Laboratory at Guangzhou Women and Children's Medical Center for assistance with radiological and laboratory examinations.

\section{References}

1 Jan de Beur SM \& Levine MA. Molecular pathogenesis of hypophosphatemic rickets. Journal of Clinical Endocrinology and Metabolism 200287 2467-2473. (https://doi.org/10.1210/ jcem.87.6.8688)

2 Pavone V, Testa G, Gioitta Iachino S, Evola FR, Avondo S \& Sessa G. Hypophosphatemic rickets: etiology, clinical features and treatment. European Journal of Orthopaedic Surgery and Traumatology 201525 221-226. (https://doi.org/10.1007/s00590-014-1496-y)

3 Gaucher C, Walrant-Debray O, Nguyen TM, Esterle L, Garabédian M $\&$ Jehan F. PHEX analysis in 118 pedigrees reveals new genetic clues in hypophosphatemic rickets. Human Genetics 2009125 401-411. (https://doi.org/10.1007/s00439-009-0631-z)

4 Holm IA, Nelson AE, Robinson BG, Mason RS, Marsh DJ, Cowell CT \& Carpenter TO. Mutational analysis and genotype-phenotype correlation of the PHEX gene in X-linked hypophosphatemic rickets. Journal of Clinical Endocrinology and Metabolism 200186 3889-3899. (https://doi.org/10.1210/jcem.86.8.7761)

5 Tyynismaa H, Kaitila I, Näntö-Salonen K, Ala-Houhala M \& Alitalo T. Identification of fifteen novel PHEX gene mutations in Finnish patients with hypophosphatemic rickets. Human Mutation 200015 383-384. (https://doi.org/10.1002/(SICI)10981004(200004)15:4<383::AID-HUMU18>3.0.CO;2-\#)

6 Ruppe MD, Brosnan PG, Au KS, Tran PX, Dominguez BW \& Northrup H. Mutational analysis of PHEX, FGF23 and DMP1 in a cohort of patients with hypophosphatemic rickets. Clinical Endocrinology 201174 312-318. (https://doi.org/10.1111/j.13652265.2010.03919.x)

7 Beck-Nielsen SS, Brock-Jacobsen B, Gram J, Brixen K \& Jensen TK. Incidence and prevalence of nutritional and hereditary rickets in southern Denmark. European Journal of Endocrinology 2009160 491-497. (https://doi.org/10.1530/EJE-08-0818)

8 Endo I, Fukumoto S, Ozono K, Namba N, Inoue D, Okazaki R, Yamauchi M, Sugimoto T, Minagawa M, Michigami T et al. Nationwide survey of fibroblast growth factor 23 (FGF23)-related hypophosphatemic diseases in Japan: prevalence, biochemical data and treatment. Endocrine Journal 201562 811-816. (https://doi. org/10.1507/endocrj.EJ15-0275)

9 Rafaelsen S, Johansson S, Ræder H \& Bjerknes R. Hereditary hypophosphatemia in Norway: a retrospective population-based study of genotypes, phenotypes, and treatment complications. European Journal of Endocrinology 2016174 125-136. (https://doi. org/10.1530/EJE-15-0515)

10 Quinlan C, Guegan K, Offiah A, Neill RO, Hiorns MP, Ellard S, Bockenhauer D, Hoff WV \& Waters AM. Growth in PHEX-associated $\mathrm{X}$-linked hypophosphatemic rickets: the importance of early treatment. Pediatric Nephrology 201227 581-588. (https://doi. org/10.1007/s00467-011-2046-z)

11 Cho HY, Lee BH, Kang JH, Ha IS, Cheong HI \& Choi Y. A clinical and molecular genetic study of hypophosphatemic rickets in children. Pediatric Research 200558 329-333. (https://doi.org/10.1203/01. PDR.0000169983.40758.7B)

12 Luo S, Huang W, Xia Q, Xia Y, Du Q, Wu L \& Duan R. Cryptic FMR1 mosaic deletion in a phenotypically normal mother of a boy with fragile X syndrome: case report. BMC Medical Genetics 201415125. (https://doi.org/10.1186/s12881-014-0125-2)

13 Slavin TP, Lazebnik N, Clark DM, Vengoechea J, Cohen L, Kaur M, Konczal L, Crowe CA, Corteville JE, Nowaczyk MJ et al. Germline mosaicism in Cornelia de Lange syndrome. American Journal of Medical Genetics: Part A 2012 158A 1481-1485. (https://doi. org/10.1002/ajmg.a.35381)

14 Lin Y, Xu A, Zeng C, Cheng J, Li N, Niu H, Liu L \& Li X. Somatic and germline FOXP3 mosaicism in the mother of a boy with IPEX syndrome. European Journal of Immunology 201848 885-887. (https:// doi.org/10.1002/eji.201747445) 
15 Walton RJ \& Bijvoet OL. Nomogram for derivation of renal threshold phosphate concentration. Lancet 19752 309-310. (https://doi. org/10.1016/s0140-6736(75)92736-1)

16 Takeda E, Yamamoto H, Nashiki K, Sato T, Arai H \& Taketani Y. Inorganic phosphate homeostasis and the role of dietary phosphorus. Journal of Cellular and Molecular Medicine 20048 191-200. (https://doi.org/10.1111/j.1582-4934.2004.tb00274.x)

17 Liu S, Tang W, Fang J, Ren J, Li H, Xiao Z \& Quarles LD. Novel regulators of FGF23 expression and mineralization in Hyp bone. Molecular Endocrinology 200923 1505-1518. (https://doi.org/10.1210/ me.2009-0085)

18 Martin A, Liu S, David V, Li H, Karydis A, Feng JQ \& Quarles LD. Bone proteins PHEX and DMP1 regulate fibroblastic growth factor FGF23 expression in osteocytes through a common pathway involving FGF receptor (FGFR) signaling. FASEB Journal 201125 2551-2562. (https://doi.org/10.1096/fj.10-177816)

19 Igaki JM, Yamada M, Yamazaki Y, Koto S, Izawa M, Ariyasu D, Suzuki E, Hasegawa H \& Hasegawa Y. High iFGF23 level despite hypophosphatemia is one of the clinical indicators to make diagnosis of XLH. Endocrine Journal 201158 647-655. (https://doi.org/10.1507/ endocrj.k10e-257)

20 Andrukhova O, Slavic S, Smorodchenko A, Zeitz U, Shalhoub V, Lanske B, Pohl EE \& Erben RG. FGF23 regulates renal sodium handling and blood pressure. EMBO Molecular Medicine 20146 744-759. (https://doi.org/10.1002/emmm.201303716)

21 Clausmeyer S, Hesse V, Clemens PC, Engelbach M, Kreuzer M, Becker-Rose P, Spital H, Schulze E \& Raue F. Mutational analysis of the PHEX gene: novel point mutations and detection of large deletions by MLPA in patients with X-linked hypophosphatemic rickets. Calcified Tissue International 200985 211-220. (https://doi. org/10.1007/s00223-009-9260-8)

22 Jonsson KB, Zahradnik R, Larsson T, White KE, Sugimoto T, Imanishi Y, Yamamoto T, Hampson G, Koshiyama H, Ljunggren O et al. Fibroblast growth factor 23 in oncogenic osteomalacia and X-linked hypophosphatemia. New England Journal of Medicine 2003 348 1656-1663. (https://doi.org/10.1056/NEJMoa020881)

23 Weber TJ, Liu S, Indridason OS \& Quarles LD. Serum FGF23 levels in normal and disordered phosphorus homeostasis. Journal of Bone and Mineral Research 200318 1227-1234. (https://doi.org/10.1359/ jbmr.2003.18.7.1227)

24 Li SS, Gu JM, Yu WJ, He JW, Fu WZ \& Zhang ZL. Seven novel and six de novo PHEX gene mutations in patients with hypophosphatemic rickets. International Journal of Molecular Medicine 201638 1703-1714. (https://doi.org/10.3892/ijmm.2016.2796)

25 Morey M, Castro-Feijóo L, Barreiro J, Cabanas P, Pombo M, Gil M, Bernabeu I, Díaz-Grande JM, Rey-Cordo L, Ariceta G et al. Genetic diagnosis of X-linked dominant hypophosphatemic Rickets in a cohort study: tubular reabsorption of phosphate and 1,25(OH)2D serum levels are associated with PHEX mutation type. BMC Medical Genetics 201112 116. (https://doi.org/10.1186/1471-2350-12-116)

26 Mumm S, Huskey M, Cajic A, Wollberg V, Zhang F, Madson KL, Wenkert D, McAlister WH, Gottesman GS, Whyte MP et al. PHEX 3'-UTR c. ${ }^{*} 231 \mathrm{~A}>\mathrm{G}$ near the polyadenylation signal is a relatively common, mild, American mutation that masquerades as sporadic or X-linked recessive hypophosphatemic rickets. Journal of Bone and Mineral Research 201530 137-143. (https://doi.org/10.1002/ jbmr.2307)

27 Goji K, Ozaki K, Sadewa AH, Nishio H \& Matsuo M. Somatic and germline mosaicism for a mutation of the PHEX gene can lead to genetic transmission of X-linked hypophosphatemic rickets that mimics an autosomal dominant trait. Journal of Clinical Endocrinology and Metabolism 200691 365-370. (https://doi.org/10.1210/jc.2005-1776)

28 Weng C, Chen J, Sun L, Zhou ZW, Feng X, Sun JH, Lu LP, Yu P \& Qi M. A de novo mosaic mutation of PHEX in a boy with hypophosphatemic rickets. Journal of Human Genetics $2016 \mathbf{6 1}$ 223-227. (https://doi.org/10.1038/jhg.2015.133)

29 Yang M, Kim J, Yang A, Jang J, Jeon TY, Cho SY \& Jin DK. A novel de novo mosaic mutation in PHEX in a Korean patient with hypophosphatemic rickets. Annals of Pediatric Endocrinology and Metabolism 201823 229-234. (https://doi.org/10.6065/ apem.2018.23.4.229)

30 Cao Y, Tokita MJ, Chen ES, Ghosh R, Chen T, Feng Y, Gorman E, Gibellini F, Ward PA, Braxton A et al. A clinical survey of mosaic single nucleotide variants in disease-causing genes detected by exome sequencing. Genome Medicine 201911 48. (https://doi. org/10.1186/s13073-019-0658-2)

Received 25 June 2019

Revised version received 1 October 2019

Accepted 28 October 2019 